\title{
RECORDING CULTURAL HERITAGE USING TERRESTRIAL LASERSCANNING - DEALING WITH THE SYSTEM, THE HUGE DATASETS THEY CREATE AND WAYS TO EXTRACT THE NECESSARY DELIVERABLES YOU CAN WORK WITH
}

\author{
E.Christofori ${ }^{\text {a, } J . ~ B i e r w a g e n ~}{ }^{\mathrm{a}}$
}

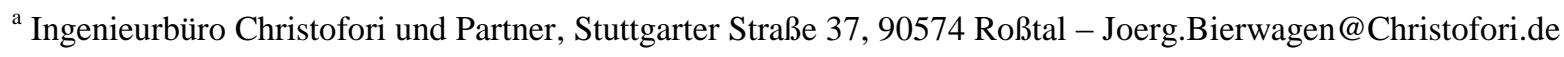

KEY WORDS: Terrestrial Laserscanning, TLS, Pointcloud, Managing, Processing, Datasets, Deliverables

\begin{abstract}
:
Recording Cultural Heritage objects using terrestrial laserscanning becomes more and more popular over the last years. Since terrestrial Laserscanning System (TLS) Manufacturers have strongly increased the amount and speed of data captured with a single scan at each system upgrade and cutting down system costs the use of TLS Systems for recording cultural heritage is an option for recording worth to think about beside traditional methods like Photogrammetric. TLS Systems can be a great tool for capturing complex cultural heritage object within a short amount of time beside the traditional methods but can be a nightmare to handle for further process if not used right while capturing.
\end{abstract}

Furthermore TLS Systems still have to be recognized as survey equipment, even though some of the manufactures promote them as everyday tool. They have to be used in an intelligent way having in mind the clients and the individual cultural objects needs. Thus the efficient way to use TLS Systems for data recording becomes a relevant topic to deal with the huge Amount of data the Systems collect while recording.

Already small projects can turn into huge Pointcloud Datasets that End user, like Architects or Archaeologist neither can't deal with as their technical equipment doesn't fit the requirements of the Dataset nor do they have the software tools to use the Data as the current software tools still are high prized. Even the necessary interpretation of the Dataset can be a tough task if the people who have to work on with the Pointcloud aren't educated right in order to understand TLS and the results it creates

The use of TLS Systems has to have in mind the project requirements of the individual Heritage Object, like the required accuracy, standards for Levels of Details (e.g. "Empfehlungen für die Baudokumentation, Günther Eckstein, Germany), the required kind of Deliverables (Visualization, 2D Drawings, True Deformation Drawings, 3D Models, BIM or 4D - Animations) as well as the Projects budget, restrictions and special conditions of the object. And if it's used in the right way TLS will fulfil all request and furthermore create additional recording, deliverable and financial benefit.

Christofori und Partner is working with TLS Systems on cultural heritage Objects since 2005 trying to optimize the use of these systems (even in combining different systems like TLS, photogrammetric or new techniques) as well as creating useable deliverables for the Clients (Owner, Conservator, Designers and the Public) they can work on with.

\section{Data Capturing using TLS - General Remark}

TLS Measuring Systems have improved enormous over the last 5 years. The Manufacturer increased the Speed of capture, Density of Capture and Accuracy of a single Pointcloud the Scanner Systems create when using it. Beside the system sizes and system weights decreased while - on first glance - the application and use of the system got much easier.

This made the systems interesting for various groups of professions beside Surveyors - who started working with the Systems at the beginning - like Architects and Archaeologist. And last but not least - also on first glance - the Costs for implementing TLS to the companies cut down with general system prices decreasing.

Some of the manufacturers even started promoting terrestrial Laserscanning as an everyday tool that anyone can use like a digital still camera on any kind of object.
"Just click the button and a nice Pointcloud will appear within a short time..." In reality it isn't that easy!

Capturing a still image with a digital still camera is more than just pressing the trigger when you want to get decent information from the image - beside some overall color information. You need to take in mind image section you need to focus on, setup the tripod correctly, light situation, object shadowing, control aperture, focal distance, etc. before you will capture an image that is worth to be taken for further use in applying it to the documentation of an heritage object.

With terrestrial 3D Laserscanning systems it's the same. The application of terrestrial 3D Laserscanning is much more - if you want to get more than just a "Pretty Image" out of it.

TLS Systems still have to be regarded as Survey Equipment that have to be used having survey standards, setups and workflows 
in mind as well as the individual Object and its surrounding and the purpose the Laserscan is made for!

\section{TLS Basics recording cultural heritage - from a Users View}

\section{a.) Purpose of data Capture}

When thinking about applying TLS Systems for recording cultural object it's necessary to consider the purpose of the required recording and the deliverables that need to be created from the recording.

In general the main purpose for using TLS system on the digital documentation of cultural heritage is to capture the as built geometry of the Object. It's the main benefit of TLS Systems as - if it's used right - it can capture the overall geometry of an object with all its deformation in high accuracy within a short amount of time.

This geometry captured and saved in a Pointcloud can be the starting point for the further documentation and investigation of the cultural object. In this context TLS has to be regarded as a tool in the toolbox in order to solve the purpose of the data capture.

It's not about TLS and the Pointcloud - it's about the Deliverables that are required for fulfilling the objects needs.

Useful deliverables created from a Pointcloud can be:

The Pointcloud itself for Geometry Backup

As TLS Systems capture geometry in $\mathrm{X}, \mathrm{Y}, \mathrm{Z}$ coordinates, reflective laser energy intensity and -depending on the systems or post processing applyColor Information in RGB Format, the Geometry can be stored as digital ASCII-Files for future reuse and geometry backup in any digital Archive. ASCII-Files will be possible to reread by any software system at any time other than any manufacture made individual Data format. If required these ASCII can even be printed or save on microfiche and stored in traditional Cultural Heritage Archives.

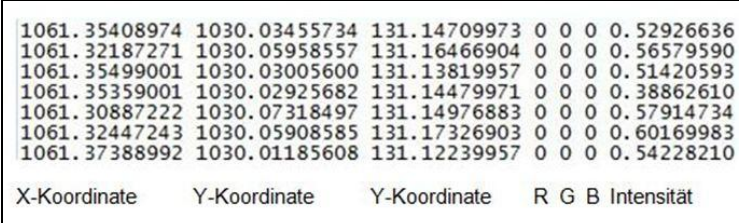

Sample Dataset Excerpt of a Pointcloud

- $\quad$ The Pointcloud itself for Visualization Purpose

Using special software tools pointclouds can be used to create visualizations of the recorded object. For geometry research, Education, Marketing, Funding, etc. Visualization Deliverables as Still Images or Videos can be created. Beside also scaled Orthoimages can be created that can be used as base for further work

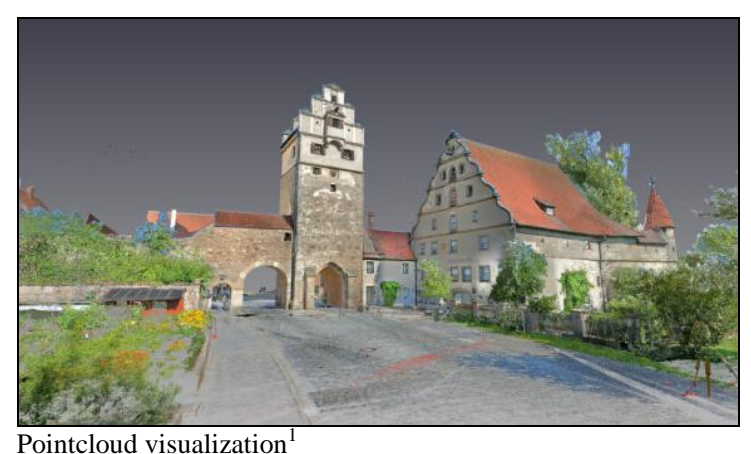

2D CAD Drawings created from the Pointcloud

Dense Pointclouds created from TLS Systems often cause tremendous problems in working with it. Pointcloud Datasets often turn out in hundreds of Gigabyte of stored data that require special computer power to perform tasks on it. So the use of the Pointcloud turns down significant on the end-users if the huge data sets need extra power and money to spend on in order to work with it.

Thus 2D CAD Drawings might turn out to be the best way to work with the Data captured by TLS Systems regarding the individual cultural objects needs, as users are used to these kinds of Deliverables and even can use them for further on site documentation.

Creating 2D CAD Drawings from a Pointcloud requires knowledge in the field of the cultural object recorded. The TLS System itself captures any geometry within the system range - nonetheless what kind of it is. When trying to create CAD- Drawings from the data, it's necessary to understand the Pointcloud and the way things are shown. The Data needs to be interpreted in order to show the required Deliverables. E.G. Parts of the Object might be shadowed in the captured Pointcloud or shown in a way that somebody might not be used to look at it. 2D CAD Drawings as Deliverables have to "speak" the speech of the professions of the users.

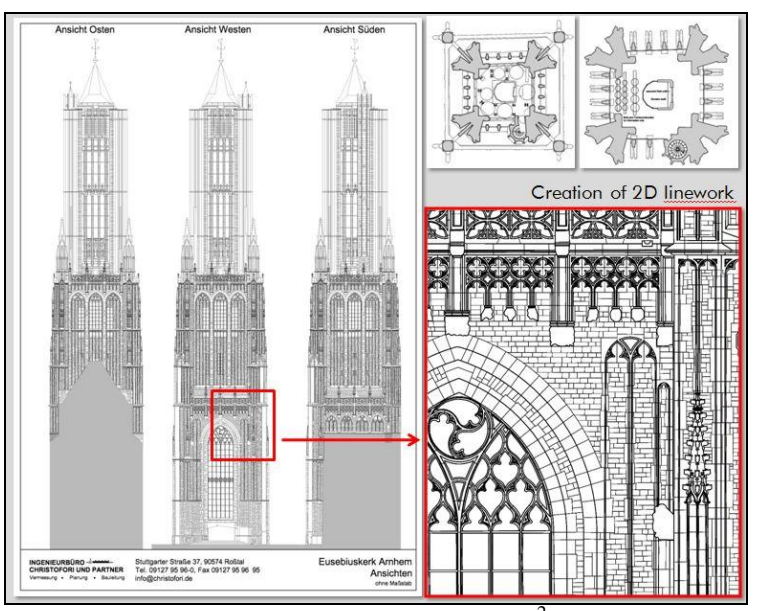

2D-Façade Drawings created from a Pointcloud ${ }^{2}$

\footnotetext{
1 Pointcloud Stadtmühle Dinkelsbühl, captured by Christofori und Partner, Roßtal, Germany

2 Eusebiuskerk Arnhem, Netherlands. Pointcloud and Deliverable Creation by Christofori und Partner, Roßtal, Germany
} 
Architectural As-Built Drawings should be created using architectural standards for heritage object documentations (e.g. "Empfehlungen für die Baudokumentation, Günther Eckstein" as standard in Germany) as archaeological site documentations should be shown in a way that Archaeologists are used to.

Without having that in mind useful Deliverables can't be created from a Pointcloud.

2D-Cad Drawings can differ in detailing level and accuracy depending on the Objects need. The Range of CAD Drawing Deliverables varies from simple overall drawings to true deformation drawings showing the object in high detail. Depending on the level of detail required to be shown in the drawings the time Assumption in creating them can raise extremely and putting up the costs in object documentation in heavy numbers.

Thus when thinking of creating 2D Drawings from a Pointcloud the detail level has play the major role in creating them - in order to fulfill the objects needs and have an efficient, cost-effective Documentation in Mind.

\section{D Models and BIM}

Dense pointclouds can be turned into 3d Models using reveres engineering software tools known from the engineering industries. 3D Models as Deliverables can be used in order to reproduce parts or an overall cultural object that might be in endangered or need to be replaced. E.g. damaged façade elements captured by TLS Systems can be transformed into a 3D Model and then be reproduced for replacing.

Modeling a Pointcloud requires expensive Software tools, various steps and reveres modeling skills until a useable result is created. The Pointcloud has to be transferred into a triangulated Mesh which then can be transferred into BSpline Nurbs models that can be used to reproduce on modern CNC molding cutter machines.

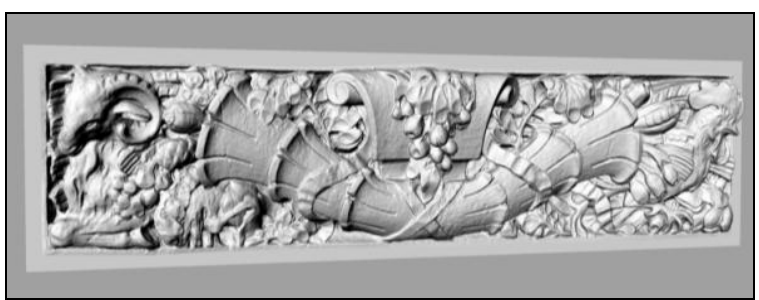

3D Model reconstructed from Pointcloud

3D models created from the Pointcloud can also be turned into BIM Models that Architects and Engineers can use for further design work. Creating models in that kind of direction hast to have in mind the current restrictions on creating the individual parts of the object with true deformation. Not all things can be shown absolutely correctly right now with the current BIM Tools.

3 3D Facade Detail Reconstruction based on Pointcloud, Vienna, Austria, Laserscan and Remodelling by Christofori und Partner, Roßtal, Germany

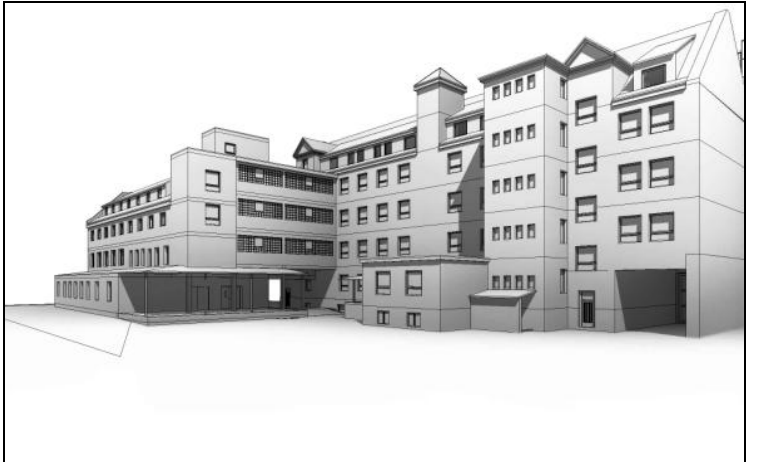

3D Model as Base for BIM Work ${ }^{4}$

- Virtual Reconstruction and 4D Animations

Pointclouds can be used as base for virtual reconstructions of former conditions of a cultural object. Combining Pointcloud true deformation Geometry information and other historic materials Reconstruction of the objects can be created and used for Education, etc. Adding a timeline in an Animation these reconstructions can show the development of a cultural object over the time.

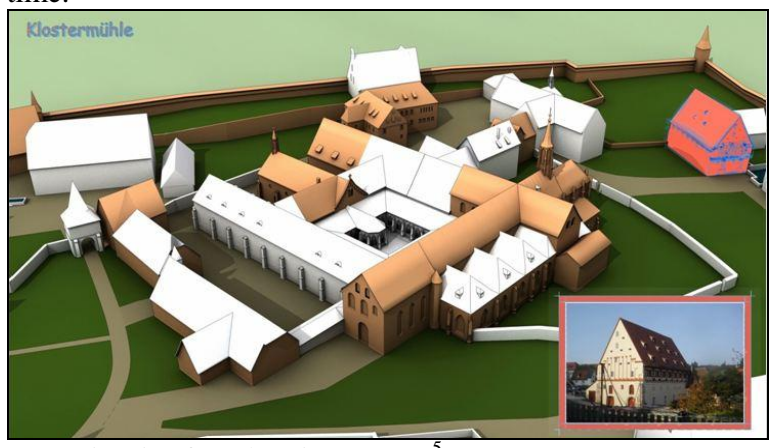

4D- Animation for Education purpose ${ }^{5}$

\section{b.) Object and Site Requirements}

Applying TLS Systems on cultural objects also requires ex ante settlement of the individual Object and site Requirements when recording the object. E.g. the Object materials can interfere with the laser systems causing problems in Data Capture. Well know for that kind of troubles are Materials like Glass, shiny Metal surfaces (also Gold) and dark, mostly black object surfaces. But also assumed ordinary materials like stone surfaces can cause problems as abrasiveness or other surface conditions can result in unusual Laser reflections and biased Pointcloud results.

Also the site conditions are worth and necessary to take in mind when applying TLS recording to a cultural object. In general cultural objects - talking about buildings in special - are not on an open plain but are part of a complex city design, which can causes restrictions on the Data Capture.

People and Cars crossing the Areas to be capture can create problems or cause special capture time periods; small streets might result in the necessity to set up a second Scanning level at higher locations in order to capture the object in full range. Or

${ }^{4}$ Hennigwerke Erlangen, Germany, 3D Model created from Pointcloud as Base for Building Information Model (BIM)

5 4D Animation Cloister Heilsbronn, Germany based on Pointcloud and historic material. Created by Christofori und Partner in Cooperation with Virtuelle-Archaeologie.de 
even scaffoldings might be needed to capture the required parts of the Object.

These entire things might turn into extra time and costs in order to capture the object and will also turn in to additional Data that needs to be processed to create the required deliverables. Thus a good site and object knowledge is mandatory if TLS should be used in a cost effective way.

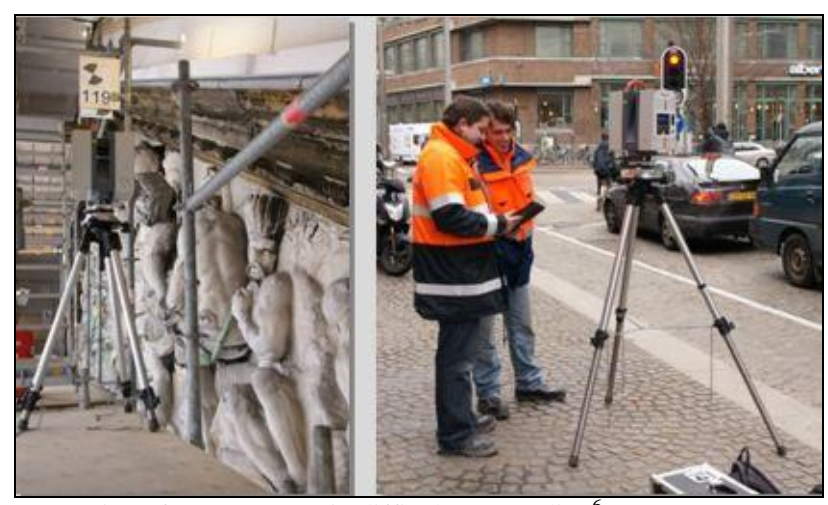

Impression of Data Capture in difficult surrounding ${ }^{6}$

\section{c.) TLS System Requirements}

Depending on the individual Object the TLS System itself has a bunch of requirements to be taken in mind when applying it. As stated earlier it has to be recognized as survey equipment. That means that traditional survey standards have to be taken in mind when setting up the scanning campaign around the object and preparing the required target positioning in order to bring together all individual scan that have been created.

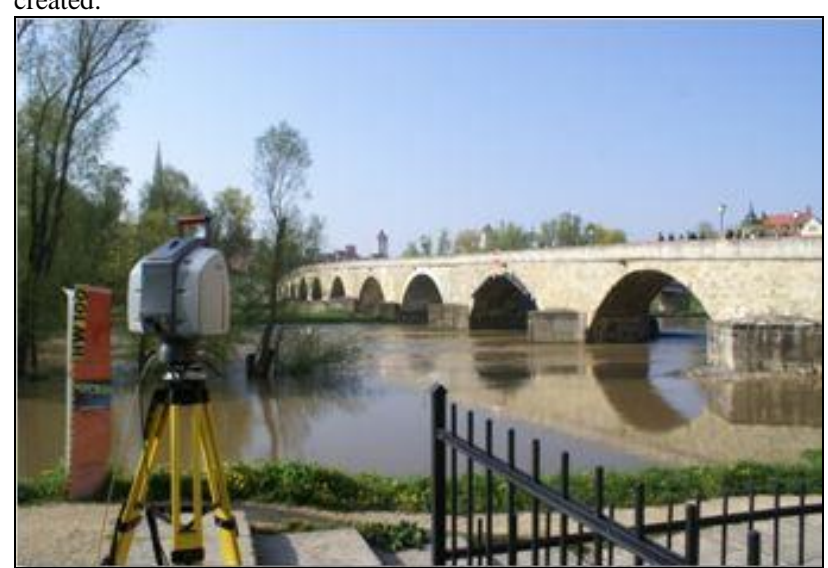

Time of Flight system used for capturing long distance object

Also the scanner decision itself shall be consider whether a Time of Flight measuring system is used on the object or a phase based measurement TLS-System is used. Both systems differ in real measurement distances, density of captured Pointcloud, accuracy and noise in the Pointcloud the produce. The given information by the Manufacturers can be only used as a guideline - not as absolute true statements. Especially the announced measurement distanced turn out be much less - if you want to work with an accurate and dense Pointcloud.

For the individual capture itself it's also very important to think about the required Pointcloud density. All scanners offer a wide bunch of scanning densities from raw preview scans to highly dense datasets.

${ }^{6}$ Data Capture in Amsterdam at the Koninklijk Paleis op de Dam, Christofori und Partner

${ }^{7}$ Laserscan capture of world heritage site Stone Bridge, Regensburg in 2008
The more dense the data gets, the Storage size for the captured Amount of data increases extremely causing delays in processing and creating deliverables as well as the capture time itself increases up to half an hour per single scan. Not always the extreme high dense Pointcloud is required and thus capture and processing time can be cut down when choosing the right parameter having the object requirements in mind.

As not all TLS Scanning systems have a color capture option included in the system itself thinking about the necessity to capture RGB Color Code information might be worth to think about in order to cut down capture times on site.

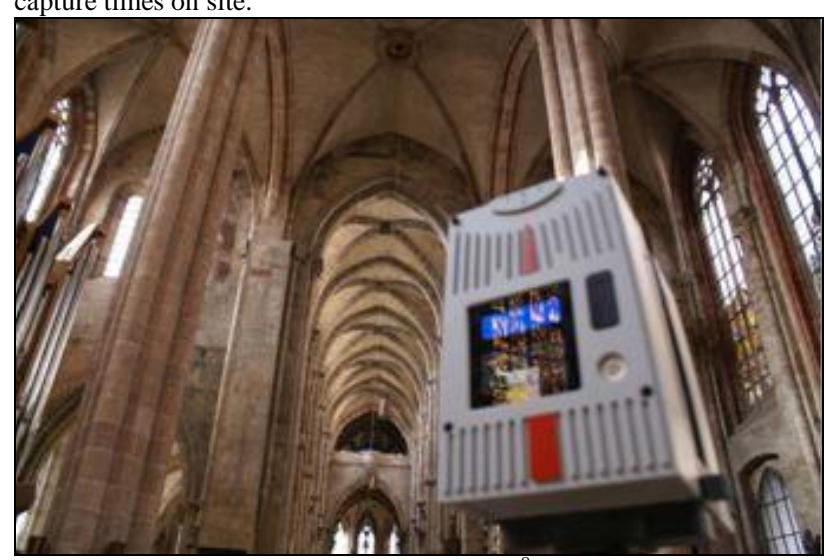

Phase based System used at interior scanning ${ }^{8}$

In order to secure accuracy of the overall Pointcloud after processing the individual scans and combining them using the overlapping targets of all scans according to our opinion it's necessary to setup a control network that will help and prove the overall accuracy of captured data.

Especially if you capture an object you cannot finish within a work day or you need to come back to object weeks or even years later, the control network created regarding widely recognized survey standards will be your secured base you can rely on at any time and continue with the capture at any time.

\section{d.) Soft- and Hardware Requirements}

As stated earlier the data captured by TLS Systems causes enormous amounts of Data to be stored and processed. Projects captured with TLS Systems can run into normal storage amounts starting by $25-50 \mathrm{~GB}$ of Data and going up to $750 \mathrm{~GB}$ to $1 \mathrm{~TB}$ or even more when working on big projects.

Thus big storage units, Backup Systems and also Workstations that can handle the huge amount of data are irrevocable necessity a company has to deal with, when thinking about applying 3D Laserscanning on cultural objects.

Even worse the software market for working with Pointcloud datasets has to be regarded. Although TLS Manufacturers produce their own software packages its necessary to use a bunch of other software tools for the individual Deliverables required to create. Up to now there is not THE one solution that fulfills all requirements.

At least 3-4 different software packages are required if deliverables like stated earlier shall be created in excellent quality. Using different software packages right now means exporting and importing Data from one Package to another. This is time consuming and is causing delays in the work progress. Even though a standardized Import-/Export Format .E57 was established at least two years ago it's still not a fully functional standard as it is not yet implemented in all tools in the right way, not working stable within the tools and not accepted by the users. Thus each tool has its special format it can work with at its best and unfortunately it's differing from tool to tool especially when transferring the data from one tool to another.

\footnotetext{
${ }^{8}$ Laserscan of St. Sebald Church, Nürnberg, 2009
} 
Visual Samples of Dataresults created from TLS by Christofori und Partner

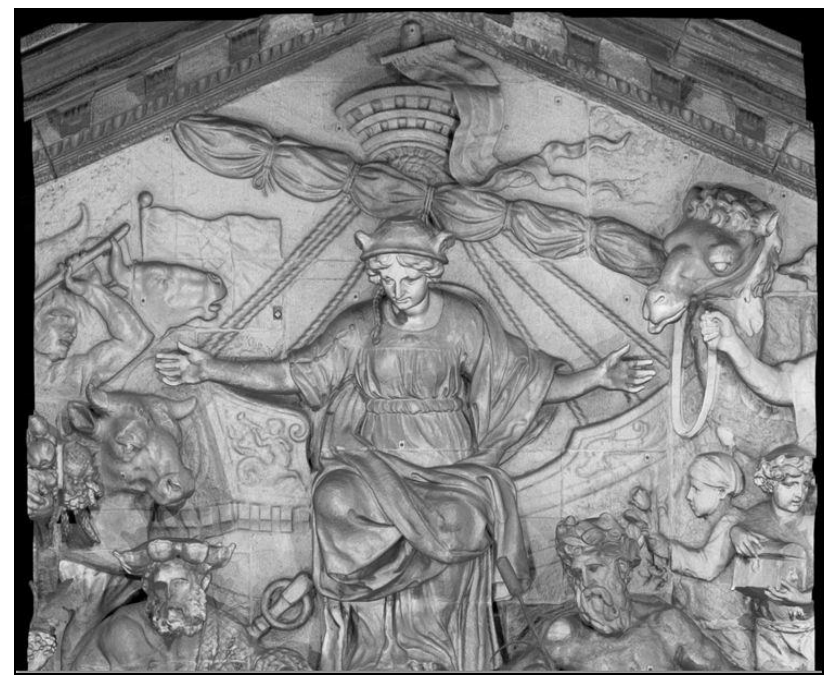

Tympanum Capture at the Koniklijk Paleis, Amsterdam

Detail of Pointcloud - Data Capture for Geometry Backup
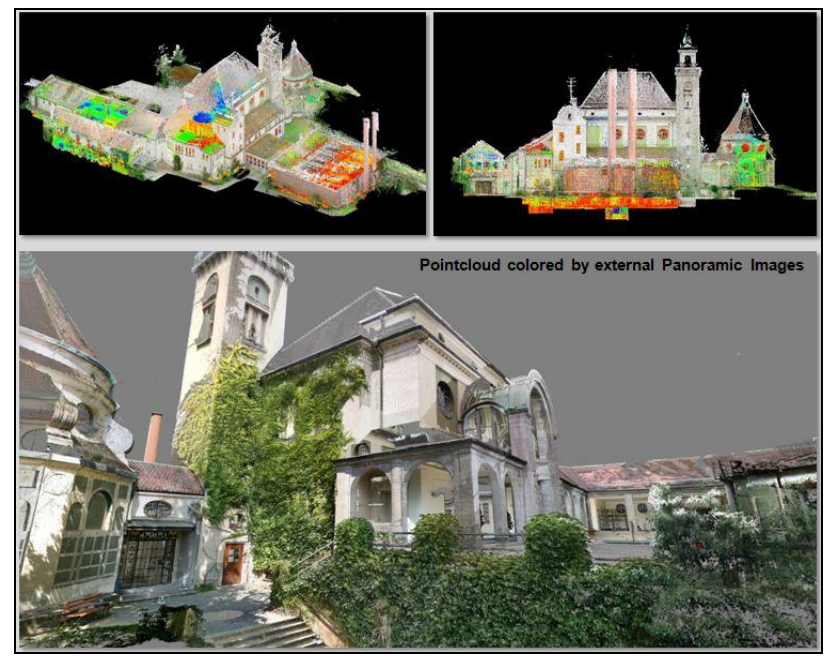

Historic Crematory, Nürnberg, National Landmark

Pointcloud Visualization

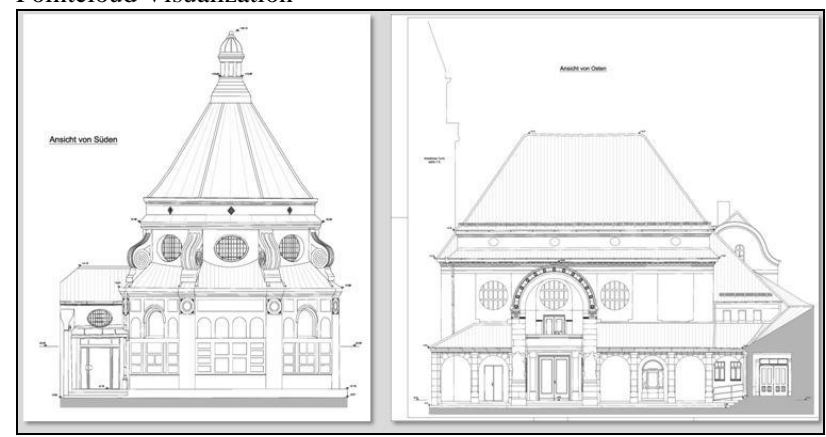

Historic Crematory, Nürnberg, National Landmark 2D CAD Elevations created (Samples)

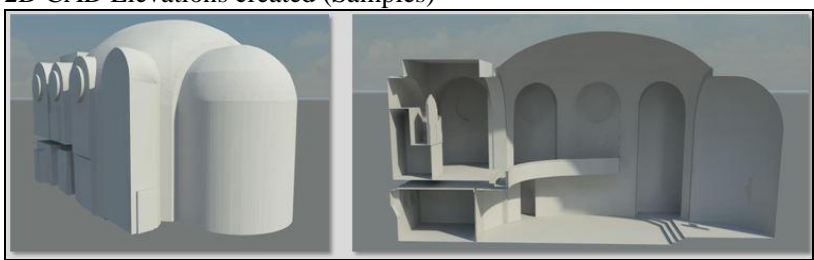

Historic Crematory, Nürnberg, National Landmark

3D Model of Building Part for Acoustic Redesign

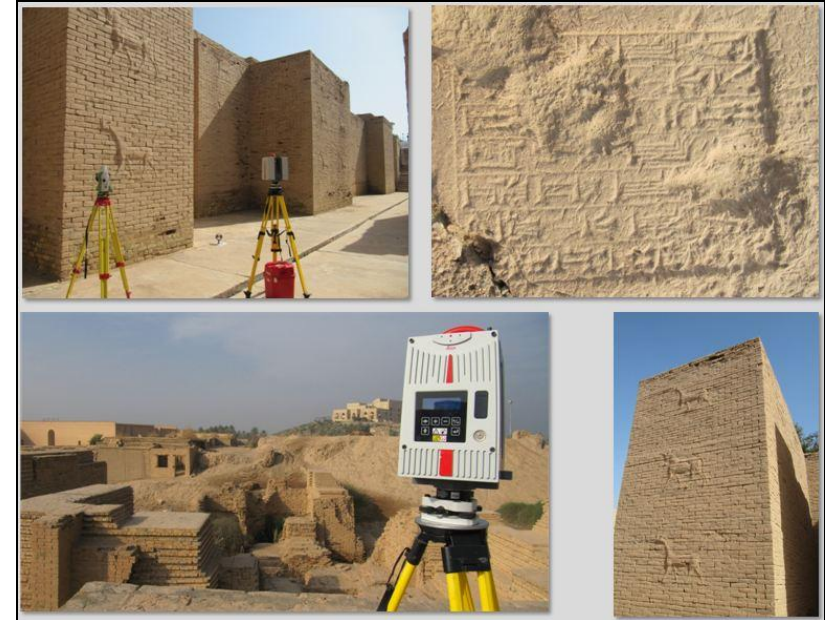

TLS Laserscan at Babylon, Iraq

Current As-Built Documentation for Reconstruction

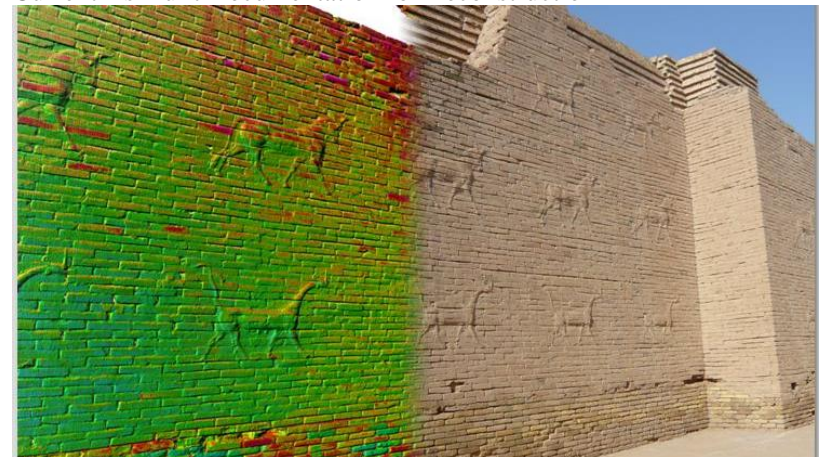

TLS Laserscan at Babylon, Iraq

Façade Detail Pointcloud

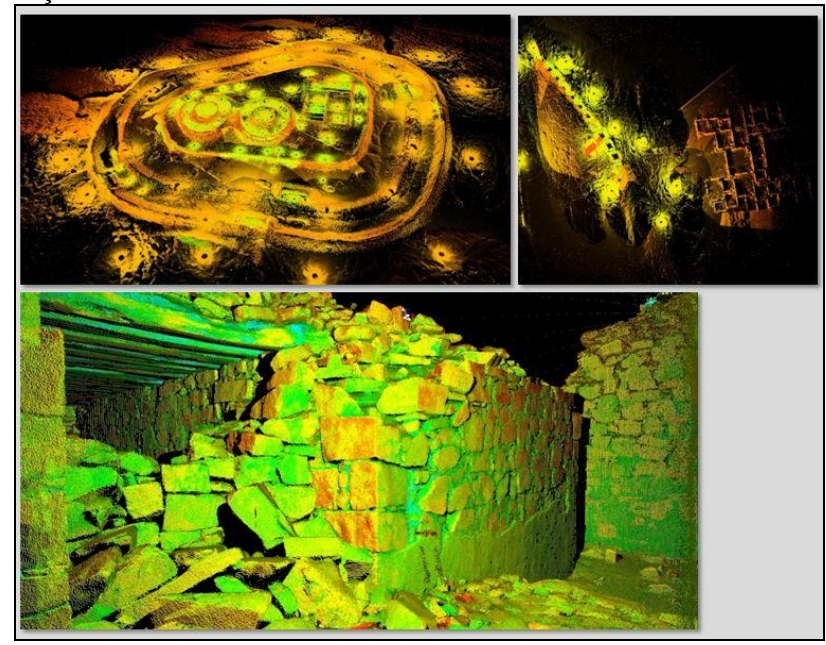

TLS Laserscan at Archaeological Site, Chanquillo, Peru Excerpts of Laserscan Capture

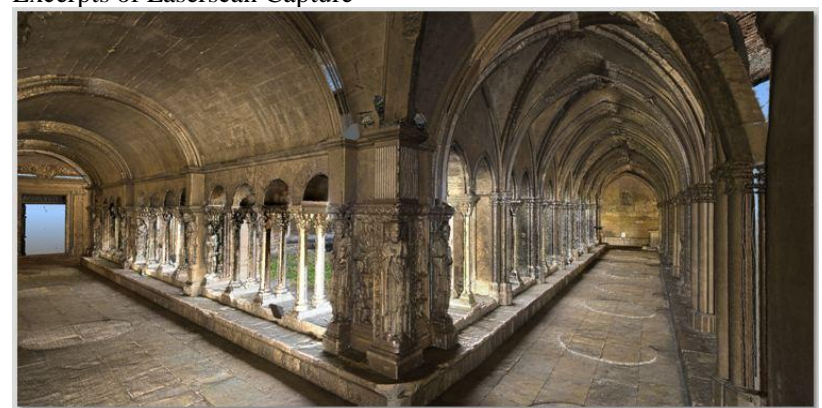

Cloister St. Trophime, Arles, Frances

TLS Laserscan with HDR Image Application - Pointcloud View showing roman and gothic construction parts of the cloister 


\section{Future of TLS Systems in capturing cultural Objects}

TLS has to be considered as to considered as state of art technology in digital geometry capture of cultural objects. The use has to be applied in an intelligent way regarding the various restrictions and requirements it needs to fulfill. It's working stable and it is producing precise geometry results that are hard to capture with other system. According to our opinion the technique used in TLS is on its peak. Developments by the manufacturers are limited on even higher data capture rates and smaller hardware.

The future of the system in the next years is bright when it's used in the right way and people are educated right in using it.

Upcoming new systems like small handheld like capturing system based on photogrammetric functionality that create pointclouds might be the follow up systems in some years and even might replace the use of TLS Systems in certain fields of work where it's a good solution right now completely. First consumer market products like the Dotprodcut $3 \mathrm{~d}^{9}$ Handscanner show of the way already.

\footnotetext{
${ }^{9}$ Dotproduct3d.com
} 